\title{
WARING'S PROBLEM FOR BEATTY SEQUENCES AND A LOCAL TO GLOBAL PRINCIPLE
}

\author{
William D. BANKS \\ Department of Mathematics \\ University of Missouri \\ Columbia, MO 65211 USA \\ bankswd@missouri.edu \\ Ahmet M. GüLOĞLU \\ Department of Mathematics \\ Bilkent University \\ 06800 Bilkent, Ankara, TURKEY \\ guloglua@fen.bilkent.edu.tr \\ Robert C. VAughan \\ Department of Mathematics \\ Pennsylvania State University \\ University Park, PA 16802-6401 USA \\ rvaughan@math.psu.edu
}

April 17, 2013

\begin{abstract}
In this paper, we investigate in various ways the representation of a large natural number $N$ as a sum of $s$ positive $k$-th powers of numbers from a fixed Beatty sequence. Inter alia, a very general form of the local to global principle is established in additive number theory. Although the proof is very short, it depends on a deep theorem of M. Kneser. There are numerous applications.
\end{abstract}




\section{Introduction}

The initial motivation for the work described in this memoir was the investigation of a variant of Waring's problem for Beatty sequences. In the process, however, a fundamental version of the local to global principle was established.

Given a set $\mathcal{A}$ of positive integers, the lower asymptotic density of $\mathcal{A}$ is the quantity

$$
\underline{\mathbf{d}}(\mathcal{A})=\liminf _{X \rightarrow \infty} \frac{\# \mathcal{A}(X)}{X},
$$

where $\mathcal{A}(X)=\mathcal{A} \cap[1, X]$. For any natural number $s$, we denote the $s$-fold sumset of $\mathcal{A}$ by

$$
s \mathcal{A}=\underbrace{\mathcal{A}+\cdots+\mathcal{A}}_{s \text { copies }}=\left\{a_{1}+\cdots+a_{s}: a_{1}, \ldots, a_{s} \in \mathcal{A}\right\} \text {. }
$$

The following very general form of the local to global principle has many applications in additive number theory.

Theorem 1. Suppose that there are numbers $s_{1}, s_{2}$ such that

(i) For all $s \geqslant s_{1}$ and $m, n \in \mathbb{N}$, the sumset $s \mathcal{A}$ has at least one element in the arithmetic progression $n \bmod m$;

(ii) The sumset $s_{2} \mathcal{A}$ has positive lower asymptotic density, i.e., $\underline{\mathbf{d}}\left(s_{2} \mathcal{A}\right)>0$.

Then, there is a number $s_{0}$ with the property that for any $s \geqslant s_{0}$ the sumset $s \mathcal{A}$ contains all but finitely many natural numbers.

Although the proof of Theorem 1 is very short (see $\S 2$ below), it relies on a deep and remarkable theorem of M. Kneser; see Halberstam and Roth [4, Chapter I, Theorem 18].

Theorem 1 has several interesting consequences. The following result (proved in $\S 3$ ) provides an affirmative answer in many instances to the question as to whether a given set of primes $\mathcal{P}$ is an asymptotic additive basis for $\mathbb{N}$.

Theorem 2. Let $\mathcal{P}$ be a set of prime numbers with

$$
\liminf _{X \rightarrow \infty} \frac{\# \mathcal{P}(X)}{X / \log X}>0
$$

Suppose that there is a number $s_{1}$ such that for all $s \geqslant s_{1}$ and $m, n \in \mathbb{N}$, the congruence

$$
p_{1}+\cdots+p_{s} \equiv n \quad(\bmod m)
$$

has a solution with $p_{1}, \ldots, p_{s} \in \mathcal{P}$. Then, there is a number $s_{0}$ with the property that for any $s \geqslant s_{0}$ the equation

$$
p_{1}+\cdots+p_{s}=N
$$

has a solution with $p_{1}, \ldots, p_{s} \in \mathcal{P}$ for all but finitely many natural numbers $N$. 
In 1770, Waring [17] asserted without proof that every natural number is the sum of at most four squares, nine cubes, nineteen biquadrates, and so on. In 1909, Hilbert [5] proved the existence of an $s_{0}(k)$ such that for all $s \geqslant s_{0}(k)$ every natural number is the sum of at most $s_{0}(k)$ positive $k$-th powers. The following result (proved in $\S 3$ ), which we deduce from Theorem 1, can be used to obtain many variants of the Hilbert-Waring theorem.

Theorem 3. Let $k \in \mathbb{N}$, and let $\mathcal{B}$ be a set of natural numbers with $\underline{\mathbf{d}}(\mathcal{B})>0$. Suppose that there is a number $s_{1}$ such that for all $s \geqslant s_{1}$ and $m, n \in \mathbb{N}$, the congruence

$$
b_{1}^{k}+\cdots+b_{s}^{k} \equiv n \quad(\bmod m)
$$

has a solution with $b_{1}, \ldots, b_{s} \in \mathcal{B}$. Then, there is a number $s_{0}$ with the property that for any $s \geqslant s_{0}$ the equation

$$
b_{1}^{k}+\cdots+b_{s}^{k}=N
$$

has a solution with $b_{1}, \ldots, b_{s} \in \mathcal{B}$ for all but finitely many natural numbers $N$.

Our work in the present paper was originally motivated by a desire to establish a variant of the Hilbert-Waring theorem with numbers from a fixed Beatty sequence. More precisely, for fixed $\alpha, \beta \in \mathbb{R}$ with $\alpha>1$, we studied the problem of representing every sufficiently large natural number $N$ as a sum of $s$ positive $k$-th powers chosen from the non-homogeneous Beatty sequence defined by

$$
\mathcal{B}_{\alpha, \beta}=\{n \in \mathbb{N}: n=\lfloor\alpha m+\beta\rfloor \text { for some } m \in \mathbb{Z}\}
$$

Beatty sequences appear in a variety of apparently unrelated mathematical settings, and the arithmetic properties of these sequences have been extensively explored in the literature. In the case that $\alpha$ is irrational, the Beatty sequence $\mathcal{B}_{\alpha, \beta}$ is distributed evenly over the congruence classes of any fixed modulus. As the congruence

$$
x_{1}^{k}+\cdots+x_{s}^{k} \equiv n \quad(\bmod m)
$$

admits an integer solution for all $m, n \in \mathbb{N}$ provided that $s$ is large enough (this follows from the Hilbert-Waring theorem but can be proved directly using Lemmas 2.13 and 2.15 of Vaughan [11] and the Chinese Remainder Theorem; see also Davenport [2, Chapter 5]), it follows that the congruence condition of Theorem 3 is easily satisfied. Since we also have $\underline{\mathbf{d}}\left(\mathcal{B}_{\alpha, \beta}\right)=\alpha^{-1}>0$, Theorem 3 yields the following corollary.

Corollary 1. Fix $\alpha, \beta \in \mathbb{R}$ with $\alpha>1$, and suppose that $\alpha$ is irrational. Then, there is a number $s_{0}$ with the property that for any $s \geqslant s_{0}$ the equation

$$
b_{1}^{k}+\cdots+b_{s}^{k}=N
$$

has a solution with $b_{1}, \ldots, b_{s} \in \mathcal{B}_{\alpha, \beta}$ for all but finitely many natural numbers $N$. 
Of course, the value of $s_{0}$ depends on $\alpha$ and a priori could be inordinately large for general $\alpha$. However, by utilising the power of the Hardy-Littlewood method we obtain the asymptotic formula for the number of solutions and show the existence of some solutions for a reasonably small value of $s_{0}$ that depends only on $k$.

Theorem 4. Fix $\alpha, \beta \in \mathbb{R}$ with $\alpha>1$, and suppose that $\alpha$ is irrational. Suppose further that $k \geqslant 2$ and that

$$
s \geqslant \begin{cases}2^{k}+1 & \text { if } 2 \leqslant k \leqslant 5, \\ 57 & \text { if } k=6, \\ 2 k^{2}+2 k-1 & \text { if } k \geqslant 7 .\end{cases}
$$

Then, the number $R(N)$ of representations of $N$ as a sum of $s$ positive $k$-th powers of members of the Beatty sequence $\mathcal{B}_{\alpha, \beta}$ satisfies

$$
R(N) \sim \alpha^{-s} \Gamma(1+1 / k)^{s} \Gamma(s / k)^{-1} \mathfrak{S}(N) N^{s / k-1} \quad(N \rightarrow \infty),
$$

where $\mathfrak{S}(N)$ is the singular series in the classical Waring's problem.

By [11, Theorems 4.3 and 4.6] the singular series $\mathfrak{S}$ satisfies $\mathfrak{S}(N) \asymp 1$ for the permissible values of $s$ in the theorem.

The lower bound demands on $s$ can be significantly reduced by asking only for the existence of solutions for all large $N$.

Theorem 5. Fix $\alpha, \beta \in \mathbb{R}$ with $\alpha>1$, and suppose that $\alpha$ is irrational. Then, there is a function $H(k)$ which satisfies

$$
H(k) \sim k \log k \quad(k \rightarrow \infty)
$$

such that if $k \geqslant 2$ and $s \geqslant H(k)$, then every sufficiently large $N$ can be represented as a sum of $s$ positive $k$-th powers of members of the Beatty sequence $\mathcal{B}_{\alpha, \beta}$.

In the interests of clarity of exposition, we have made no effort to optimise the methods employed. Certainly many refinements are possible. For instance, in the range $5 \leqslant k \leqslant 20$ it would be possible to give explicit values for the function $H(k)$ by extracting the relevant bounds for Lemma 2 below from Vaughan and Wooley [13, 14, 15, 16], and doubtless the exponent $4 k$ of $S(\vartheta)$ can be replaced by 2 with some reasonable effort.

\section{$1.1 \quad$ Notation}

The notation $\|x\|$ is used to denote the distance from the real number $x$ to the nearest integer, that is,

$$
\|x\|=\min _{n \in \mathbb{Z}}|x-n| \quad(x \in \mathbb{R}) .
$$


We denote by $\{x\}$ the fractional part of $x$. We put $\mathbf{e}(x)=e^{2 \pi i x}$ for all $x \in \mathbb{R}$. Throughout the paper, we assume that $k$ and $n$ are natural numbers with $k \geqslant 2$.

For any finite set $S$, we denote by $\# S$ the number of elements in $S$.

In what follows, any implied constants in the symbols $\ll$ and $O$ may depend on the parameters $\alpha, \beta, k, s, \varepsilon, \eta$ but are absolute otherwise. We recall that for functions $F$ and $G$ with $G \geqslant 0$ the notations $F \ll G$ and $F=O(G)$ are equivalent to the statement that the inequality $|F| \leqslant c G$ holds for some constant $c>0$. If $F \geqslant 0$ also, then $F \gg G$ is equivalent to $G \ll F$. We also write $F \asymp G$ to indicate that $F \ll G$ and $G \ll F$.

\section{The proof of Theorem 1}

Let $\delta_{s}=\underline{\mathbf{d}}(s \mathcal{A})$ for each $s$. Note that hypothesis $(i i)$ implies that $\delta_{s}>0$ for all $s \geqslant s_{2}$. We now suppose that $s=\max \left(s_{1}, s_{2}\right)$ and appeal to Kneser's theorem in the form given in $\left[4, \S 1\right.$, Theorem 18]; we conclude that for each $t=1,2, \ldots$, either (case 1) $\delta_{t s} \geqslant t \delta_{s}$ or (case 2) there is a set of integers $\mathcal{A}^{\prime}$ which is worse than $\mathcal{A}_{t s}$ and degenerate $\bmod g^{\prime}$ for some positive integer $g^{\prime}$ (here, worse means that $\mathcal{A}_{t s} \subset \mathcal{A}^{\prime}$ and that the sets $\mathcal{A}_{t s}$ and $\mathcal{A}^{\prime}$ coincide from some point onwards, and degenerate $\bmod g^{\prime}$ means that $\mathcal{A}^{\prime}$ is a union of residue classes to some modulus $g^{\prime}$ ). Since $\delta_{s}>0$ and $\delta_{t s} \leqslant 1$ it follows that case 2 must occur if $t$ is large enough. Let $t$ be fixed with this property. As $t s \geqslant t s_{1} \geqslant s_{1}$, from the definition of $s_{1}$ we see that for arbitrary $h, m$ and $n$ the residue class $h+m g^{\prime} \bmod n g^{\prime}$ intersects $\mathcal{A}_{t s}$. By a judicious choice of $m$ and $n$ there will be a sufficiently large element of $\mathcal{A}_{t s}$ in the residue class $h+m g^{\prime} \bmod n g^{\prime}$, and this element will also lie in $\mathcal{A}^{\prime}$. Clearly, this

element also lies in the residue class $h \bmod g^{\prime}$. Since $h$ is arbitrary and $\mathcal{A}^{\prime}$ is degenerate $\bmod g^{\prime}$, it follows that $\mathcal{A}^{\prime}=\mathbb{Z}$. But $\mathcal{A}_{t s}$ and $\mathcal{A}^{\prime}$ coincide from some point onwards, and therefore, $\mathcal{A}_{t s}$ contains every sufficiently large positive integer.

\section{The proofs of Theorems 2 and 3}

For any set $\mathcal{S} \subset \mathbb{N}$, let $R_{s}(n ; \mathcal{S})$ be the number of $s$-tuples $\left(a_{1}, \ldots, a_{s}\right)$ with entries in $\mathcal{S}$ for which $a_{1}+\cdots+a_{s}=n$.

To prove Theorem 3 we specialise the set $\mathcal{A}$ in Theorem 1 to be the set of $k$-th powers of elements of $\mathcal{B}$. Let $\mathcal{A}^{*}$ denote the set of $k$-th powers of all natural numbers, and suppose that $s>2^{k}$. Using Theorem 2.6 and (2.19) of [11] we have

$$
R_{s}(n ; \mathcal{A}) \leqslant R_{s}\left(n ; \mathcal{A}^{*}\right) \ll n^{s / k-1} .
$$

Also, the hypothesis $\underline{\mathbf{d}}(\mathcal{B})>0$ implies that

$$
\# \mathcal{A}(N / s)=\# \mathcal{B}\left((N / s)^{1 / k}\right) \gg(N / s)^{1 / k} \gg N^{1 / k}
$$


provided that $(N / s)^{1 / k}$ is no smaller than the least element of $\mathcal{B}$. Thus, if we write $A_{s}(N)=$ $\#(s \mathcal{A} \cap[1, N])$, then for such $N$ we have

$$
N^{s / k} \ll(\# \mathcal{A}(N / s))^{s} \leqslant \sum_{n=1}^{N} R_{s}(n ; \mathcal{A}) \ll A_{s}(N) N^{s / k-1} .
$$

We can conclude the proof by observing that the congruence condition in Theorem 1 is immediate from that in Theorem 3.

Theorem 2 can be established in the same way. It suffices to show that if $\mathcal{P}^{*}$ is the set of all primes, then for some $s$ we have

$$
R_{s}\left(n ; \mathcal{P}^{*}\right) \ll n^{s-1}(\log 2 n)^{-s} \quad(n \in \mathbb{N}) .
$$

When $s=3$ this is immediate from Theorem 3 and (3.15) in Chapter 3 of [11], and it would also follow rather easily from a standard application of sieve theory, although none of the standard texts establish the required result explicitly. Alternatively, the standard sieve bound

$$
R_{2}\left(n ; \mathcal{P}^{*}\right) \ll \frac{n^{2}}{\varphi(n)(\log 2 n)^{2}} \quad(n \in \mathbb{N})
$$

(which follows from Halberstam and Richert [3, Corollary 2.3.5], for example) and a simple application of Cauchy's inequality show that $\underline{\mathbf{d}}(2 \mathcal{P})>0$.

\section{The generating functions}

The rest of this memoir is devoted to the study of the special case of sums of $k$-th powers of members of a Beatty sequence via the Hardy-Littlewood method. Let

$$
\mathcal{B}(P)=\left\{n \in \mathcal{B}_{\alpha, \beta}: n \leqslant P\right\} \quad \text { and } \quad \mathcal{A}(P, R)=\{n \leqslant P: p \mid n \Longrightarrow p \leqslant R\},
$$

and put

$$
\begin{aligned}
S(\vartheta) & =\sum_{n \in \mathcal{B}(P)} e\left(\vartheta n^{k}\right), & T(\vartheta) & =\sum_{n \leqslant P} e\left(\vartheta n^{k}\right), \\
U(\vartheta) & =\sum_{n \in \mathcal{A}(P, R) \cap \mathcal{B}(P)} e\left(\vartheta n^{k}\right), & V(\vartheta) & =\sum_{n \in \mathcal{A}(P, R)} e\left(\vartheta n^{k}\right),
\end{aligned}
$$

Lemma 1. Suppose that $t$ satisfies

$$
t \geqslant \begin{cases}3 & \text { if } k=2, \\ 2^{k-1} & \text { if } 3 \leqslant k \leqslant 5, \\ 56 & \text { if } k=6, \\ 2 k^{2}+2 k-2 & \text { if } k \geqslant 7 .\end{cases}
$$


If $F$ is one of $S, U$ or $V$, then

$$
\int_{0}^{1}|F(\vartheta)|^{2 t} d \vartheta \leqslant \int_{0}^{1}|T(\vartheta)|^{2 t} d \vartheta \ll P^{2 t-k} .
$$

Proof. When $k=2$ the bound on $\int_{0}^{1}|T(\vartheta)|^{2 t} d \vartheta$ follows from a standard application of the Hardy-Littlewood method, when $k=3$ from Vaughan [8, Theorem 2], when $k=4$ or 5 from Vaughan [9], when $k=6$ from Boklan [1], and when $k \geqslant 7$ from Wooley [18, Corollary 4] and a routine application of the Hardy-Littlewood method. The proof is completed by interpreting each integral as the number of solutions of the diophantine equation

$$
x_{1}^{k}+\cdots+x_{t}^{k}=x_{t+1}^{k}+\cdots+x_{2 t}^{k}
$$

with the $x_{j}$ lying in $\mathcal{B}(P), \mathbb{N} \cap[1, P], \mathcal{A}(P, R) \cap \mathcal{B}(P)$ or $\mathcal{A}(P, R)$, respectively.

Lemma 2. There is a number $\eta>0$ and a function $H_{1}(k)$ such that

$$
H_{1}(k) \sim k \log k \quad(k \rightarrow \infty)
$$

with the property that whenever $2 t \geqslant H_{1}(k)$ and $R=P^{\eta}$ we have

$$
\int_{0}^{1}\left|S(\vartheta)^{4 k} U(\vartheta)^{2 t}\right| d \vartheta \leqslant \int_{0}^{1}\left|T(\vartheta)^{4 k} V(\vartheta)^{2 t}\right| d \vartheta \ll P^{2 t+3 k} .
$$

Proof. In view of Lemma 1 , it can be supposed that $k \geqslant k_{0}$ for a suitable $k_{0}$. According to [11, Theorem 12.4] we have

$$
\int_{0}^{1}|V(\vartheta)|^{2 s} d \vartheta \ll P^{\lambda_{s}+\varepsilon}
$$

where

$$
\lambda_{s}=2 s-k+k \exp (1-2 s / k) .
$$

Let $\mathfrak{m}$ denote the set of real numbers $\vartheta \in[0,1]$ such that if $|\vartheta-a / q| \leqslant q^{-1} P^{3 / 4-k}$ with $(a, q)=1$, then $q>P^{3 / 4}$, and let $\mathfrak{M}=[0,1] \backslash \mathfrak{m}$. Then, by Vaughan [10, Theorem 1.8] we have

$$
\sup _{\vartheta \in \mathfrak{m}}|V(\vartheta)| \ll P^{1-\sigma_{k}+\varepsilon}
$$

where

$$
\sigma_{k}=\max _{\substack{n \in \mathbb{N} \\ n \geqslant 2}} \frac{1}{4 n}\left(1-(k-2)(1-1 / k)^{n-2}\right) .
$$

Note that

$$
\sigma_{k} \sim \frac{1}{4 k \log k} \quad(k \rightarrow \infty)
$$

We now put

$$
s=\left\lfloor\frac{1}{2} k \log k+k \log \log k\right\rfloor+1 \quad \text { and } \quad t=s+k .
$$


Then,

$$
\int_{\mathfrak{m}}|V(\vartheta)|^{2 t} d \vartheta \ll P^{2 t-k+\mu_{k}+\varepsilon}
$$

where

$$
\mu_{k}=k \exp (1-2 s / k)-2 k \sigma_{k}<e(\log k)^{-2}-2 k \sigma_{k}<0
$$

provided that $k>k_{0}$. Hence

$$
\int_{\mathfrak{m}}\left|T(\vartheta)^{4 k} V(\vartheta)^{2 t}\right| d \vartheta \ll P^{2 t+3 k} .
$$

By the methods of $[11$, Chapter 4$]$ we also have

$$
\int_{\mathfrak{M}}\left|T(\vartheta)^{4 k} V(\vartheta)^{2 t}\right| d \vartheta \ll P^{2 t} \int_{\mathfrak{M}}|T(\vartheta)|^{4 k} d \vartheta \ll P^{2 t+3 k}
$$

and the lemma is proved.

In what follows, we denote

$$
S(q, a)=\sum_{m=1}^{q} e\left(a m^{k} / q\right) \quad \text { and } \quad I(\phi)=\int_{0}^{P} e\left(\phi x^{k}\right) d x .
$$

Lemma 3. Suppose that $\alpha$ is irrational. Then, for every real number $P \geqslant 1$ there is a number $Q=Q(P)$ such that

(i) $Q \leqslant P^{1 / 2}$

(ii) $Q \rightarrow \infty$ as $P \rightarrow \infty$;

(iii) Let $\mathfrak{m}$ denote the set of real numbers $\vartheta$ with the property that $q>Q$ whenever the inequality $|\vartheta-a / q| \leqslant Q q^{-1} P^{-k}$ holds with $(a, q)=1$. Then,

$$
S(\vartheta) \ll P Q^{-1 / k} \quad(\vartheta \in \mathfrak{m})
$$

(iv) If $q \leqslant Q,|\vartheta-a / q| \leqslant Q q^{-1} P^{-k}$, and $(a, q)=1$, then

$$
S(\vartheta)=\alpha^{-1} q^{-1} S(q, a) I(\vartheta-a / q)+O\left(P Q^{-1 / k}\right) .
$$

Proof. Since $\alpha \notin \mathbb{Q}$, there is at most one pair of integers $m, n$ such that $n=\alpha m+\beta$ and at most one pair such that $n=\alpha m+\beta-1$. For any other value of $n$ we have

$$
n=\lfloor\alpha m+\beta\rfloor \text { for some } m \quad \Longleftrightarrow \quad 1-\alpha^{-1}<\left\{\alpha^{-1}(n-\beta)\right\}<1 \text {. }
$$


Let $\Psi(x)=x-\lfloor x\rfloor-\frac{1}{2}$ for all $x \in \mathbb{R}$; then $\Psi$ is periodic with period one, and for $x \in[0,1)$ we have

$$
\alpha^{-1}+\Psi(x)-\Psi\left(x+\alpha^{-1}\right)= \begin{cases}1 & \text { if } 1-\alpha^{-1}<x<1, \\ 0 & \text { if } 0<x<1-\alpha^{-1}, \\ \frac{1}{2} & \text { if } x=0 \text { or } x=1-\alpha^{-1} .\end{cases}
$$

Consequently,

$$
S(\vartheta)=\alpha^{-1} T(\vartheta)+\sum_{n \leqslant P}\left(\Psi\left(\alpha^{-1}(n-\beta)\right)-\Psi\left(\alpha^{-1}(n-\beta+1)\right)\right) e\left(\vartheta n^{k}\right)+O(1) .
$$

Now let

$$
T(\vartheta, \phi)=\sum_{n \leqslant P} e\left(\vartheta n^{k}+\phi n\right)
$$

and

$$
W(\phi)=\sum_{n \leqslant P} \min \left\{1, H^{-1}\left\|\alpha^{-1} n-\phi\right\|^{-1}\right\}
$$

where $H$ is a positive parameter to be determined below. By Montgomery and Vaughan [6, Lemma D.1] we have

$$
\begin{gathered}
S(\vartheta)=\alpha^{-1} T(\vartheta)-\sum_{0<|h| \leqslant H} \frac{e\left(\alpha^{-1}(1-\beta) h\right)-e\left(-\alpha^{-1} \beta h\right)}{2 \pi i h} T\left(\vartheta, \alpha^{-1} h\right) \\
+O\left(1+W\left(\alpha^{-1} \beta\right)+W\left(\alpha^{-1}(\beta-1)\right)\right) .
\end{gathered}
$$

Choose $r=r(P)$ maximal and $b$ so that

$$
(b, r)=1, \quad\left|\alpha^{-1}-b / r\right| \leqslant r^{-2} \quad \text { and } \quad r^{2}\left|\alpha^{-1}-b / r\right|^{-1} \leqslant P^{1 / 4} .
$$

This is always possible if $P$ is large enough. Indeed, by Dirichlet's theorem on diophantine approximation, or by the theory of continued fractions, there are infinitely many coprime pairs $b, r$ that satisfy the first inequality, and at least one of the pairs will satisfy the second inequality if $P$ is sufficiently large. Moreover, the two inequalities together imply that $r \leqslant P^{1 / 16}$, so the maximal $r$ exists. Note that $r=r(P)$ tends to infinity as $P \rightarrow \infty$ since $\alpha$ is irrational. Let $\xi=\alpha^{-1} r^{2}-b r$, choose $c$ so that $|\phi r-c| \leqslant \frac{1}{2}$, put $\eta=\phi r-c$, and for every $n \leqslant P$ write $n=u r+v$ with $-r / 2<v \leqslant r / 2$ and $0 \leqslant u \leqslant 1+P / r$. For any given $u$, let $w$ be an integer closest to $u \xi$, and put $\kappa=u \xi-w$. Then,

$$
W(\phi)=\sum_{u, v} \min \left\{1, H^{-1}\left\|\alpha^{-1}(u r+v)-\phi\right\|^{-1}\right\}
$$

Moreover,

$$
\alpha^{-1}(u r+v)-\phi=u b+\frac{v b+w-c}{r}+\frac{\kappa}{r}+\frac{v \xi}{r^{2}}-\frac{\eta}{r}
$$


and for any given $u$ we have

$$
\left\|\alpha^{-1}(u r+v)-\phi\right\| \geqslant\left\|\frac{v b+w-c}{r}\right\|-\frac{3}{2 r} .
$$

Hence the contribution to $W$ from any fixed $u$ is

$$
\ll 1+H^{-1} r \log r,
$$

and so summing over all $u$ we derive the bound

$$
W(\phi) \ll P r^{-1}+P H^{-1} \log r .
$$

The choice $H=r^{1 / 3}$ gives

$$
S(\vartheta)=\alpha^{-1} T(\vartheta)-\sum_{0<|h| \leqslant r^{1 / 3}} \frac{e\left(\alpha^{-1}(1-\beta) h\right)-e\left(-\alpha^{-1} \beta h\right)}{2 \pi i h} T\left(\vartheta, \alpha^{-1} h\right)+O\left(\operatorname{Pr}^{-1 / 4}\right) .
$$

The error term here is acceptable provided that $Q \leqslant r^{1 / 4}$.

Next, we show that the sum over $h$ is also $\ll P Q^{-1}$ provided that $Q=Q(P)$ grows sufficiently slowly. Choose $a, q$ with $(a, q)=1$ such that $|\vartheta-a / q| \leqslant q^{-1} P^{\frac{1}{2}-k}$ and $q \leqslant P^{k-\frac{1}{2}}$. Then, by [11, Lemma 2.4], when $q>P^{1 / 2}$ there is a $\delta=\delta(k)>0$ such that

$$
T(\vartheta, \phi) \ll P^{1-\delta} \quad(\phi \in \mathbb{R}) .
$$

Since $T(\vartheta)=T(\vartheta, 0)$ and $r \leqslant P^{1 / 16}$, we derive the bound

$$
S(\vartheta) \ll P^{1-\delta} \log P+P r^{-1 / 4} \ll P Q^{-1}
$$

provided that $Q \leqslant \min \left\{P^{\delta} / \log P, r^{1 / 4}\right\}$, and we are done in this case.

Now suppose that $q \leqslant P^{1 / 2}$. We have

$$
\begin{aligned}
T\left(\vartheta, \alpha^{-1} h\right) & =\sum_{m=1}^{q} e\left(a m^{k} / q\right) \sum_{\substack{n \leqslant P \\
n \equiv m(\bmod q)}} e\left((\vartheta-a / q) n^{k}+\alpha^{-1} h n\right) \\
& =q^{-1} \sum_{\frac{h q}{\alpha}-\frac{q}{2}<\ell \leqslant \frac{h q}{\alpha}+\frac{q}{2}} S(q, a, \ell) \sum_{n \leqslant P} e\left((\vartheta-a / q) n^{k}+\left(\alpha^{-1} h-\ell / q\right) n\right),
\end{aligned}
$$

where

$$
S(q, a, \ell)=\sum_{m=1}^{q} e\left(a m^{k} / q+\ell m / q\right) .
$$

Let $g$ be the polynomial

$$
g(x)=(\vartheta-a / q) x^{k}+\left(\alpha^{-1} h-\ell / q\right) x .
$$


For $0 \leqslant x \leqslant P$ and $\frac{h q}{\alpha}-\frac{q}{2}<\ell \leqslant \frac{h q}{\alpha}+\frac{q}{2}$ it is easy to verify that

$$
\left|g^{\prime}(x)\right| \leqslant k q^{-1} P^{-1 / 2}+\frac{1}{2}<\frac{3}{4}
$$

if $P$ is large enough. Hence, by Titchmarsh [7, Lemma 4.8] we see that

$$
\sum_{n \leqslant P} e\left((\vartheta-a / q) n^{k}+\left(\alpha^{-1} h-\ell / q\right) n\right)=\int_{0}^{P} e(g(x)) d x+O(1) .
$$

In the case that $\left|\alpha^{-1} h-\ell / q\right| \geqslant 1 /(2 q)$, we have

$$
\left|g^{\prime}(x)\right| \geqslant\left|\alpha^{-1} h-\ell / q\right|-k q^{-1} P^{-1 / 2} \gg\left|\alpha^{-1} h-\ell / q\right|,
$$

and therefore by [7, Lemma 4.2] the integral in (4.4) is

$$
\ll\left|\alpha^{-1} h-\ell / q\right|^{-1} \text {. }
$$

Also, we have trivially $|S(q, a, \ell)| \leqslant q$. Thus, the total contribution to $T\left(\vartheta, \alpha^{-1} h\right)$ from the numbers $\ell$ with $\left|\alpha^{-1} h-\ell / q\right| \geqslant 1 /(2 q)$ is

$$
\ll \sum_{\substack{\ell \\\left|\alpha^{-1} h-\ell / q\right| \geqslant 1 /(2 q)}}\left|\alpha^{-1} h-\ell / q\right|^{-1} \ll q \log q,
$$

and summing over $h$ with $0<|h| \leqslant r^{1 / 3}$ the overall contribution to the sum in (4.3) is

$$
\ll q \log q \cdot \log r \ll P^{3 / 4}
$$

which is acceptable.

Next, let $\ell$ be a number for which $\left|\alpha^{-1} h-\ell / q\right|<1 /(2 q)$; note that there is at most one such $\ell$ for each $h$. Since $(a, q)=1$, by [11, Theorem 7.1] we have that $S(q, a, \ell) \ll q^{1-1 / k+\varepsilon}$. Hence the total contribution to the sum in (4.3) from such an $\ell$ is $\ll q^{-1 / k+\varepsilon} P \log r$. When $q>r^{1 / 3}$ this is sufficient provided that $Q \leqslant r^{1 / 4}$. Now suppose that $q \leqslant r^{1 / 3}$. Since $\alpha$ is irrational and $r$ is large, we have $b \neq 0$ by (4.2), and we claim that $h b / r \neq \ell / q$. Indeed, suppose on the contrary that $h b q=r \ell$. Then $b \mid \ell$, and we can write $\ell=m b$, and $h q=r m$. Since $h \neq 0$, it follows that $m \neq 0$. But this is impossible since $|h| q \leqslant r^{2 / 3}$, and the claim is proved. Therefore, using (4.2) again, we have

$$
\left|\alpha^{-1} h-\ell / q\right|=\left|h b / r-\ell / q+h\left(\alpha^{-1}-b / r\right)\right| \geqslant|h b / r-\ell / q|-|h| r^{-2} \geqslant(r q)^{-1}-r^{-5 / 3} \gg(r q)^{-1} .
$$

Arguing as before, we see that $\left|g^{\prime}(x)\right| \gg(r q)^{-1}$, the integral in (4.4) is $\ll r q$, and therefore $T\left(\vartheta, \alpha^{-1} h\right) \ll q^{1-1 / k+\varepsilon} r$ for each $h$ associated with such an $\ell$; hence the total contribution to the sum in $(4.3)$ is

$$
\ll q^{1-1 / k+\varepsilon} r \log r \ll r^{4 / 3} \leqslant P^{1 / 12} .
$$


It remains only to deal with the single term

$$
\alpha^{-1} T(\vartheta)
$$

By [11, Theorem 4.1] we have

$$
\alpha^{-1} T(\vartheta)=\alpha^{-1} q^{-1} S(q, a) I(\vartheta-a / q)+O(q)
$$

and since $q \leqslant P^{1 / 2}$ the error term here is acceptable. By [11, Lemma 2.8],

$$
I(\vartheta-a / q) \ll \min \left(P,|\vartheta-a / q|^{-1 / k}\right)
$$

and by $[11$, Theorem 4.2] we have

$$
S(q, a) \ll q^{1-1 / k}
$$

Hence, if $q>Q$ or $|\vartheta-a / q|>Q /\left(q P^{k}\right)$ we see that

$$
\alpha^{-1} T(\vartheta) \ll P Q^{-1 / k}
$$

The only remaining $\vartheta$ to be considered are those for which there exist coprime integers $a, q$ with $q \leqslant Q$ and $|\vartheta-a / q| \leqslant Q q^{-1} P^{-k}$. Thus, we have shown that for all $\vartheta$ in $\mathfrak{m}$ the desired bound holds. For the remaining $\vartheta$, we have established that $(i v)$ holds as required.

For $\varphi \in \mathbb{R}$ and a parameter $A>1$ at our disposal which will eventually be chosen as a function of $\varepsilon$ (only), define

$$
\begin{aligned}
& f_{-}(\varphi)=\max \left\{0,(A+1)\left(1-2 \alpha\left\|1-\frac{1}{2 \alpha}-\varphi\right\|\right)\right\}-\max \left\{0, A-2 \alpha(A+1)\left\|1-\frac{1}{2 \alpha}-\varphi\right\|\right\}, \\
& f_{+}(\varphi)=\max \left\{0, A+1-2 \alpha A\left\|1-\frac{1}{2 \alpha}-\varphi\right\|\right\}-\max \left\{0, A\left(1-2 \alpha\left\|1-\frac{1}{2 \alpha}-\varphi\right\|\right)\right\} .
\end{aligned}
$$

Let

$$
S_{ \pm}(\vartheta)=\sum_{n \leqslant P} f_{ \pm}((n-\beta) / \alpha) e\left(\vartheta n^{2}\right)
$$

The functions $f_{ \pm}$respectively minorize and majorize the characteristic function of the set $[1-1 / \alpha, 1] \bmod 1$. Thus, following the discussion in the first paragraph of the proof of Lemma 3 , with the choice $P=N^{1 / 2}$ we have

$$
\int_{0}^{1} S_{-}(\vartheta)^{s} e(-\vartheta N) d \vartheta \leqslant R(N) \leqslant \int_{0}^{1} S_{+}(\vartheta)^{s} e(-\vartheta N) d \vartheta
$$

in the case that $k=2$. The functions $f_{ \pm}$have Fourier expansions

$$
f_{ \pm}(\varphi)=\sum_{h=-\infty}^{\infty} c_{ \pm}(h) e(h \varphi)
$$


whose coefficients are given by

$$
c_{-}(0)=\alpha^{-1}\left(1-\frac{1}{2(A+1)}\right), \quad c_{+}(0)=\alpha^{-1}\left(1+\frac{1}{2 A}\right),
$$

and for any $h \neq 0$,

$$
\begin{aligned}
& c_{-}(h)=\frac{e\left(\frac{1}{2} \alpha^{-1} h\right)(A+1) \alpha}{\pi^{2} h^{2}}\left(\cos \frac{\pi \alpha^{-1} h A}{A+1}-\cos \pi \alpha^{-1} h\right), \\
& c_{+}(h)=\frac{e\left(\frac{1}{2} \alpha^{-1} h\right) A \alpha}{\pi^{2} h^{2}}\left(\cos \pi \alpha^{-1} h-\cos \frac{\pi \alpha^{-1} h(A+1)}{A}\right) .
\end{aligned}
$$

Note that

$$
c_{ \pm}(h) \ll h^{-2} A \alpha \quad(h \neq 0) .
$$

Lemma 4. Suppose that $(a, q)=1$ and $|\vartheta q-a| \leqslant P^{-1}$. Then

$$
S_{ \pm}(\vartheta) \ll A \alpha\left(\frac{P}{\left(q+P^{2}|\vartheta q-a|\right)^{1 / 2}}+q^{1 / 2}\right) .
$$

Proof. By (4.1), (4.5) and (4.7),

$$
S_{ \pm}(\vartheta)=\sum_{h=-\infty}^{\infty} c_{ \pm}(h) e(-h \beta / \alpha) T(\vartheta, h / \alpha) .
$$

The conclusion then follows from (4.9) and Vaughan [12, Theorem 5].

Lemma 5. Suppose that $\alpha$ is irrational. Then, for every real number $P \geqslant 1$ there is a number $Q=Q(P)$ such that

(i) $Q \leqslant P^{1 / 2}$

(ii) $Q \rightarrow \infty$ as $P \rightarrow \infty$;

(iii) For any coprime integers a, $q$ with $q \leqslant Q$ and $|\vartheta-a / q| \leqslant Q q^{-1} P^{-2}$ we have

$$
S_{ \pm}(\vartheta)=c_{ \pm}(0) q^{-1} S(q, a) I(\vartheta-a / q)+O\left(P Q^{-1 / 2}\right)
$$

Proof. This can be established in the same way as Lemma 3. 


\section{The proofs of Theorems 4 and 5}

When $k>2$, Theorem 4 follows from Lemmas 1 and 3 by a routine application of the Hardy-Littlewood method.

When $k=2$, let $Q$ be as in Lemma 5. Now define

$$
\mathfrak{M}(q, a)=\left\{\vartheta:|\vartheta-a / q| \leqslant Q q^{-1} P^{-2}\right\}
$$

and let $\mathfrak{M}$ denote the union of the $\mathfrak{M}(q, a)$ with $1 \leqslant a \leqslant q \leqslant Q$ and $(a, q)=1$. Put $\mathfrak{m}=\left[Q P^{-2}, 1+Q P^{-2}\right] \backslash \mathfrak{M}$, so that $\mathfrak{m} \subset\left[Q P^{-2}, 1-Q P^{-2}\right)$. Now for any $\vartheta \in \mathfrak{m}$ we choose coprime integers $a, q$ with $1 \leqslant a \leqslant q \leqslant P$ and $|\vartheta-a / q| \leqslant q^{-1} P^{-1}$. Note that, by the definition of $\mathfrak{m}$, we have $|\vartheta-a / q|>q^{-1} P^{-1}$ when $q \leqslant Q$. By Lemma 4 , whenever $s \geqslant 5$ we have

$$
\begin{aligned}
\int_{\mathfrak{m}}\left|S_{ \pm}(\vartheta)\right|^{s} d \vartheta & \ll \sum_{q \leqslant Q} q \int_{Q q^{-1} P^{-2}}^{1 /(q P)}(A \alpha)^{s}\left(q^{-s / 2} \varphi^{-s / 2}+q^{s / 2}\right) d \varphi \\
& +\sum_{Q<q \leqslant P} q \int_{0}^{1 /(q P)}(A \alpha)^{s}\left(P^{s}\left(q+P^{2} q \varphi\right)^{-s / 2}+q^{s / 2}\right) d \varphi \\
& \ll(A \alpha)^{s} \sum_{q \leqslant Q}\left(P^{s-2} Q^{1-s / 2}+P^{-1} q^{s / 2}\right)+(A \alpha)^{s} \sum_{Q<q \leqslant P}\left(q^{1-s / 2} P^{s-2}+P^{-1} q^{s / 2}\right) \\
& \ll(A \alpha)^{s}\left(Q^{-1 / 2} P^{s-2}+P^{s / 2}\right) \ll \alpha^{-s} P^{s-2} Q^{-1 / 4} .
\end{aligned}
$$

Choosing $P=N^{1 / 2}$, a routine application of Lemma 5 shows that

$$
\int_{\mathfrak{M}} S_{ \pm}(\vartheta)^{s} e(-N \vartheta) d \vartheta=c_{ \pm}(0) \Gamma(3 / 2)^{s} \Gamma(s / 2)^{-1} \mathfrak{S}(N) N^{s / 2-1}+O\left(N^{s / 2-1} Q^{-1 / 4}\right) .
$$

Now suppose that $A=1 / \varepsilon$, where $\varepsilon$ is positive but small. Then, by (4.6) and (4.8) it follows that

$$
R(N)=\alpha^{-s} \Gamma(3 / 2)^{s} \Gamma(s / 2)^{-1} \mathfrak{S}(N) N^{s / 2-1}+O\left(\varepsilon N^{s / 2-1}\right) \quad\left(N>N_{0}(\varepsilon)\right),
$$

and this completes the proof of Theorem 4 .

To prove Theorem 5 we take $P=N^{1 / k}, R$ and $t$ as in Lemma 2 and consider the number $R(N)$ of representations of $N$ in the form

$$
N=x_{1}^{k}+\cdots+x_{4 k+1}^{k}+y_{1}^{k}+\cdots+y_{2 t}^{k}
$$

with $x_{1}, \ldots, x_{4 k+1} \in \mathcal{B}(P)$ and $y_{1}, \ldots, y_{2 t} \in \mathcal{A}(P, R) \cap \mathcal{B}(P)$. Clearly,

$$
R(N)=\int_{0}^{1} S(\vartheta)^{4 k+1} U(\vartheta)^{2 t} e(-N \vartheta) d \vartheta
$$


Let $\mathfrak{M}(q, a)$ denote the set of $\vartheta$ with $|\vartheta-a / q| \leqslant Q q^{-1} P^{-k}$, let $\mathfrak{M}$ be the union of all such intervals with $1 \leqslant a \leqslant q \leqslant Q$ and $(a, q)=1$, and put $\mathfrak{m}=\left(Q P^{-k}, 1+Q P^{-k}\right] \backslash \mathfrak{M}$. By Lemmas 2 and 3 we have

$$
\int_{\mathfrak{m}}\left|S(\vartheta)^{4 k+1} U(\vartheta)^{2 t}\right| d \vartheta \ll P^{3 k+2 t+1} Q^{-1 / k}
$$

Let

$$
Z(\vartheta)= \begin{cases}\alpha^{-1} q^{-1} S(q, a) I(\vartheta-a / q) & \text { if } \vartheta \in \mathfrak{M}(q, a), \\ 0 & \text { if } \vartheta \in \mathfrak{m} .\end{cases}
$$

Then, by $(i v)$ of Lemma 3 and a routine argument we have

$$
\int_{\mathfrak{M}} S(\vartheta)^{4 k+1} U(\vartheta)^{2 t} e(-N \vartheta) d \vartheta=\int_{Q P^{-k}}^{1+Q P^{-k}} Z(\vartheta)^{4 k+1} U(\vartheta)^{2 t} e(-N \vartheta) d \vartheta+O\left(P^{3 k+2 t+1} Q^{-1 / k}\right) .
$$

By the methods of $[11$, Chapter 4] we have

$$
\int_{Q P^{-k}}^{1+Q P^{-k}} Z(\vartheta)^{4 k+1} e(-m \vartheta) d \vartheta=\alpha^{-4 k-1} \frac{\Gamma(1+1 / k)^{4 k+1}}{\Gamma(4+1 / k)} m^{3+1 / k} \mathfrak{S}(m)+O\left(P^{3 k+1} Q^{-1 / k}\right)
$$

uniformly for $1 \leqslant m \leqslant N$, and

$$
\int_{Q P^{-k}}^{1+Q P^{-k}} Z(\vartheta)^{4 k+1} e(-m \vartheta) d \vartheta \ll P^{3 k+1} Q^{-1 / k}
$$

uniformly for $m \leqslant 0$. Here $\mathfrak{S}$ is the usual singular series associated with Waring's problem; note that $\mathfrak{S}(m) \asymp 1$. Therefore,

$$
\begin{array}{rl}
\int_{Q P^{-k}}^{1+Q P^{-k}} & Z(\vartheta)^{4 k+1} U(\vartheta)^{2 t} e(-N \vartheta) d \vartheta \\
= & \sum_{y_{1}, \ldots, y_{2 t}} \alpha^{-4 k-1} \frac{\Gamma(1+1 / k)^{4 k+1}}{\Gamma(4+1 / k)}\left(N-y_{1}^{k}-\cdots-y_{2 t}^{k}\right)^{3+1 / k} \mathfrak{S}\left(N-y_{1}^{k}-\cdots-y_{2 t}^{k}\right) \\
& +O\left(P^{3 k+2 t+1} Q^{-1 / k}\right),
\end{array}
$$

where the sum is taken over those $y_{1}, \ldots, y_{2 t} \in \mathcal{B}(P)$ with $\left(N-y_{1}^{k}-\cdots-y_{2 t}^{k}\right)^{3+1 / k}>0$. By restricting to those $y_{1}, \ldots, y_{2 t}$ that do not exceed $P /(4 t)$, one sees that

$$
R(N) \gg N^{3+1 / k+2 t / k}
$$

if $N$ is sufficiently large, and this completes the proof of Theorem 5 . 


\section{References}

[1] K. D. Boklan, 'The asymptotic formula in Waring's problem', Mathematika 41 (1994), no. $2,329-347$.

[2] H. Davenport, Analytic methods for Diophantine equations and Diophantine inequalities. Second edition. Cambridge Mathematical Library. Cambridge University Press, Cambridge, 2005.

[3] H. Halberstam and H.-E. Richert, Sieve methods. London Mathematical Society Monographs, No. 4. Academic Press, London-New York, 1974.

[4] H. Halberstam and K. F. Roth, Sequences. Second edition. Springer-Verlag, New YorkBerlin, 1983.

[5] D. Hilbert, 'Beweis für die Darstellbarkeit der ganzen Zahlen durch eine feste Anzahl $n^{\text {ter }}$ Potenzen (Waringsches Problem)', Math. Ann. 67 (1909), no. 3, 281-300.

[6] H. L. Montgomery and R. C. Vaughan, Multiplicative number theory. I. Classical theory. Cambridge Studies in Advanced Mathematics, 97. Cambridge University Press, Cambridge, 2007.

[7] E. C. Titchmarsh, The theory of the Riemann zeta-function. Second edition. The Clarendon Press, Oxford University Press, New York, 1986.

[8] R. C. Vaughan, 'On Waring's problem for cubes', J. Reine Angew. Math. 365 (1986), $122-170$.

[9] R. C. Vaughan, 'On Waring's problem for smaller exponents II', Mathematika 33 (1986), no. 1, 6-22.

[10] R. C. Vaughan, 'A new iterative method in Waring's problem', Acta Math. 162 (1989), no. 1-2, 1-71.

[11] R. C. Vaughan, The Hardy-Littlewood method. Second edition. Cambridge Tracts in Mathematics, 125. Cambridge University Press, Cambridge, 1997.

[12] R. C. Vaughan, 'On generating functions in additive number theory, I', Analytic Number Theory, Essays in Honour of Klaus Roth, 436-448, Cambridge Univ. Press, Cambridge, 2009.

[13] R. C. Vaughan and T. D. Wooley, 'Further improvements in Waring's problem, III: Eighth powers', Philos. Trans. Roy. Soc. London Ser. A 345 (1993), no. 1676, 385-396.

[14] R. C. Vaughan and T. D. Wooley, 'Further improvements in Waring's problem, II: Sixth powers', Duke Math. J. 76 (1994), no. 3, 683-710. 
[15] R. C. Vaughan and T. D. Wooley, 'Further improvements in Waring's problem', Acta Math. 174 (1995), no. 2, 147-240.

[16] R. C. Vaughan and T. D. Wooley, 'Further improvements in Waring's problem, IV: Higher powers', Acta Arith. 94 (2000), no. 3, 203-285.

[17] E. Waring, Meditationes algebraicœ. Cambridge, England, 1770.

[18] T. D. Wooley, 'Vinogradov's mean value theorem via efficient congruencing', Ann. Math., to appear. 